\title{
Hair cortisol concentration in Siberian flying squirrels is unrelated to landscape and social factors
}

\author{
Andrea Santangeli $^{1}$ (D) $\cdot$ Ralf Wistbacka $^{2} \cdot$ Chiara Morosinotto $^{3} \cdot$ Aura Raulo $^{4}$ \\ Received: 22 November 2018 / Revised: 6 May 2019 / Accepted: 11 May 2019 / Published online: 29 May 2019 \\ (C) The Author(s) 2019
}

\begin{abstract}
Intact ecosystems are being lost or modified worldwide, and many animal species are now forced to live in altered landscapes. A large amount of scientific studies have focused on understanding direct effects of habitat alterations on species occurrence, abundance, breeding success, and other life history aspects. Much less attention has been placed on understanding how habitat alterations impact on the physiology of species, e.g., via elevated chronic stress when living in an altered landscape. Here, we quantify the effects of individual age and sex, as well as effects of landscape and social factors on chronic stress of an endangered forest specialist species, the Siberian flying squirrel Pteromys volans. We collected hair samples over 2 years from across 192 flying squirrels and quantified their chronic stress response via cortisol concentrations. We then ran statistical models to relate cortisol concentrations with landscape and social factors. We show that cortisol concentrations in flying squirrels are neither affected by habitat amount and connectivity, nor by the density of conspecifics in the area. We however found that cortisol concentration was higher in adults than in pups, and in males compared with females. Lack of an effect of environmental factors on cortisol concentrations may indicate low physiological sensitivity to alterations in the surrounding environment, possibly due to low densities of predators that could induce stress in the study area. Further research should focus on possible effects of varying predator densities, alone and in interaction with landscape features, in shaping chronic stress of this and other species.
\end{abstract}

Keywords Animal physiology $\cdot$ Stress hormones $\cdot$ Land-use change $\cdot$ Conservation physiology $\cdot$ Habitat fragmentation $\cdot$ Boreal forest

\section{Introduction}

The loss, fragmentation, and degradation of habitats are major drivers of the current biodiversity crisis and species extinction

Communicated by: Matthias Waltert

Electronic supplementary material The online version of this article (https://doi.org/10.1007/s00114-019-1624-y) contains supplementary material, which is available to authorized users.

Andrea Santangeli

andrea.santangeli@helsinki.fi

1 The Helsinki Laboratory of Ornithology, Finnish Museum of Natural History, University of Helsinki, P.O. Box 17, FI-00014 Helsinki, Finland

2 Department of Ecology and Genetics, University of Oulu, P.O. Box 3000, Oulu, Finland

3 Bioeconomy Research Team, The Novia University of Applied Sciences, Raseborgsvägen 9, FI-10600 Ekenäs, Finland

4 Department of Zoology, University of Oxford, Oxford OX1 3PS, UK globally (Pimm et al. 1995). In order to tackle and reverse these drivers of threat, it is crucial to have a deep understanding of the processes and mechanisms linking species and individual responses to rapid environmental changes. Unfortunately, such understanding is still very limited for several species, and this lack of knowledge strongly reduces the effectiveness of conservation actions (Sutherland et al. 2004).

Habitat changes and disturbance can influence the behavior and physiological status of a species, ultimately affecting reproduction and population persistence (Santangeli et al. 2012). Although demographic impacts (e.g., on breeding success and survival) resulting from changes in the environment have been widely documented (Ewers and Didham 2006; Newton 1998), physiological impacts (e.g., increase in chronic stress) have only recently been considered in studies within the field of applied ecology and conservation (Creel et al. 2002; Navarro-Castilla et al. 2014; Tarjuelo et al. 2015). Vertebrates typically cope with noxious temporary stimuli by initiating a stress response (Sheriff et al. 2011). If such a stress response is activated and maintained over time due to a permanent stimulus, e.g., a change in the environment that 
becomes inhospitable to the species, the stress may become chronic, impairing individual's health with repercussions on fecundity and survival (Charbonnel et al. 2008; Rakotoniaina et al. 2017; Sapolsky et al. 2000; Sheriff et al. 2011).

Habitat loss (i.e., the reduction in forest patch size in the landscape), fragmentation (i.e., the breaking apart of forest patches thus becoming increasingly isolated), and degradation (e.g., selective logging that changes the forest structure) may force forest-dwelling species to change their behavior, cross gaps in forests and use forest edges. Crossing forest gaps and moving along forest edges may ultimately result in increased stress for forest specialist species (Brearley et al. 2012). These effects have been for example demonstrated for several arboreal squirrel species in North America and Europe (Koprowski 2005; Chen and Koprowski 2016; Mäkeläinen et al. 2016; Trapp et al. 2019). Human-induced environmental changes may also affect inter- and intraspecific interactions leading to increased predation risk (e.g., Brearley et al. 2012; Gorini et al. 2012; Smith 2012), as well as affect resource availability and competition (Ewers and Didham 2006).

Steroid hormones, particularly cortisol and corticosterone, have been used to investigate whether animals living in different environments experience different levels of chronic stress (Sheriff et al. 2011). Cortisol concentrations in feces and hair have been used as a non-invasive approach to quantify stress caused by anthropogenic changes in the environment in several vertebrates (Brearley et al. 2012; Sheriff et al. 2011). In addition to the physiological consequences for the adults, environmental stressors may also affect offspring growth and fitness through the stress perceived by breeding females (Bairos-Novak et al. 2018; Dantzer et al. 2013). Few studies have shown that females exposed to high stress during the early phase of reproduction produce fewer and smaller offspring with higher levels of steroid hormones (Sheriff et al. 2009). These transgenerational effects of stress could potentially influence population dynamics, through a decline in reproductive success (Sheriff et al. 2010).

Ultimately, anthropogenic environmental stressors, such as habitat changes, could affect animal populations both directly, through their influence on breeding adults, as well as indirectly, through transgenerational effects on the offspring. This may carry important implications for habitat management and wildlife conservation. However, we broadly lack knowledge about the effects of environmental (e.g., resource availability) and social factors (e.g., density of congeners, sex, and age) on the stress levels of many elusive animals, such as forest-dwelling nocturnal species. Many such forest specialist species are endangered, particularly in the Boreal forests of Europe and North America, largely owing to intensive forest management practices (Koprowski 2005; Santangeli et al. 2013a, b). Therefore, it is relevant to study the physiological, in addition to the demographic, response of such species to potential sources of stress in semi-natural as well as anthropic landscapes.
Herein, we contribute to fill the above knowledge gap by investigating environmental and social factors potentially associated with chronic stress, measured as hair cortisol concentration, of an endangered mammal species, the Siberian flying squirrel (Pteromys volans; hereafter flying squirrel). So far, no studies have investigated the physiology of this species in general, and particularly in relation to the potential drivers of chronic stress. Understanding how environmental changes may lead to a stress response is particularly important in endangered species, because the possible consequences of stress on local populations could lead to drastic consequences for the overall species conservation. In such cases, it is also important to adopt the least invasive sampling approach, to minimize additional stress to the individual.

Specifically, (1) we quantified whether cortisol concentrations in flying squirrel hairs vary depending on age (adults versus pups) and sex (males vs females). Because sampling was done during the reproductive season, we predict adult females to have higher cortisol levels compared with adult males, as is common among female mammals in general (Huhman et al. 2003; Nikicicz et al. 1984; Reeder and Kramer 2005; Waterhouse et al. 2017). (2) We quantified the relationship between cortisol concentrations and landscape factors, e.g., amount of suitable habitat, at different spatial scales, (3) as well as the impact of the density of other adults of the same sex in the area. We predict that cortisol concentrations would be higher in areas with lower availability of suitable habitat, in small patches and patches that are most exposed to open areas (Brearley et al. 2012), and where congener densities are highest due to increased competition (Charbonnel et al. 2008). Assessing effects of congener density is relevant because in the Siberian flying squirrel, males are smaller than females and compete for females through a scramble competition mating system (Selonen et al. 2016). Thus, high male densities may result in increased stress. Finally, (4) we quantified whether the cortisol concentrations of adult and pups flying squirrels is related to individual body condition and (5) whether cortisol concentrations of adult females correlated with cortisol concentrations and body condition of their offspring. We expect that individual in better condition would have lower levels of cortisol (Shero et al. 2015), and that cortisol concentrations of mothers would be positively correlated with cortisol concentrations of pups but negatively with pups body condition (Bairos-Novak et al. 2018).

\section{Methods}

\section{Study species and study landscape}

The flying squirrel is a nocturnal arboreal squirrel widely spread throughout the Eurasian taiga. Within the European 
Union, most of the species population occurs in the southern part of Finland (Santangeli et al. 2013a). Populations of flying squirrels in Finland are declining due to destruction of their primary habitat, layered mixed forests dominated by mature spruce (Koskimäki et al. 2014). These forests provide food resources, nesting places, and shelter. The flying squirrel is strictly arboreal, and although it can cross narrow openings, it refrains from crossing large gaps (Selonen and Hanski 2003). Large spruces are perceived to provide cover against predators and are used for storing winter food (Mäkelä 1996). Litters are reared in cavities built by the great spotted woodpecker (Dendrocopos major) but nest boxes are readily used (Koskimäki et al. 2014; Lampila et al. 2009). Adults have a strong site fidelity, with males' home range being about 60 ha while that of females is about 8 ha (Hanski et al. 2000).

Contrary to many other mammal species, female flying squirrels are larger than males (Selonen et al. 2016). Females of this species are also territorial, spending the whole life within their home range that does not overlap with that of other females (Selonen et al. 2013). The home range of males can instead overlap with that of several other males, and can include multiple female home ranges (Selonen et al. 2013). Male's movement activity increases during the breeding season, when males compete for females, and multi-male paternity can occur within the same litter (Selonen et al. 2013). The flying squirrel is globally classified as Least Concern according to the IUCN Species Red List, but within the European Union is listed in the Habitats Directive, which has been enforced through ineffective habitat protection in Finland (Jokinen et al. 2014; Santangeli et al. 2013b; Wistbacka et al. 2018).

The study area in western Finland (municipalities of Larsmo-Öja and Vaasa, see Fig. 1) is largely comprised of intensively managed boreal forests and is extensively described in Santangeli et al. (2013b) and Wistbacka et al. (2018). Most forests in the Larsmo-Öja area are privately owned and managed for timber production ending with a final clear-cut when the forest is about 60 to 80 years old. As a result, most forests in this area are younger than 80 years (Finnish Forest Research Institute 2014), with scattered patches of older forest that nowadays remain suitable for the flying squirrel to breed (Wistbacka et al. 2018). Conversely, the Vaasa area is largely dominated by urban parkland stretching along the coast-line near the city of Vaasa. This recreational area is largely composed of sparse but large trees suitable for flying squirrels to breed. The trees in this area are largely unmanaged; therefore, their average age is higher than that of the Larsmo-Öja area.

The study system is comprised of 69 study sites (forest patches suitable for breeding, see below; $n=10$ in Vaasa and 59 in Larsmo-Öja), each including 1 to 5 nest boxes (depending on the size of the patch) suitable for flying squirrels. These sites are part of a long-term study on the species ecology, conservation, and life history (Santangeli et al. 2013b; Selonen et al. 2014; Wistbacka et al. 2018).

\section{Collection of samples and body measures}

During 2015 and 2016, all nest boxes in the study area have been checked in June (corresponding to the species breeding period, i.e., when pups are about 6 weeks old) and all adults and pups have been sexed, aged, their body mass, and femur length measured (see Selonen et al. 2016). A sample of about $10 \mathrm{mg}$ of hairs was then cut from the side of the tail of each individual. Cutting was done as close as possible to the base of the hair while avoiding any risk of harming the tissue of an individual. All hair samples were cut by the same observer with the same protocol. For logistic reasons, we could not apply the clip and regrowth protocol that would allow knowing the exact time span of the hair growth as suggested by Sheriff et al. (2011). Nevertheless, the hair sampling has been carried out in a systematic and consistent way for all individuals; therefore, the resulting cortisol concentrations are comparable between individuals, as they would all relate to a similar time span of hair growth, during same time of the year. Collected hair samples were stored in a dry place at room temperature and then sent off to be analyzed in the lab for extraction of cortisol levels. Hair samples were chosen because they are indicative of chronic stress relevant for a long time period spanning weeks to a few months (i.e., the growth time of hairs; Sheriff et al. 2011), compared with, e.g., blood samples from which measured stress levels may be affected by short-term events, including handling of the animal (Desantis et al. 2016; Sheriff et al. 2011). Moreover, this method is least invasive and thus more ethically acceptable than, e.g., drawing blood samples, particularly for a species that is nationally protected and listed within the Habitats Directive of the EU. In the lab, samples were washed with $100 \%$ methanol for $2 \mathrm{~min}$. Methanol was then decanted and the dried samples were powdered in a bead mill. Next, hairs were extracted with $400 \mu \mathrm{l}$ $90 \%$ methanol and aliquots of $20 \mu \mathrm{l}$, diluted 1:2 with water, and analyzed in the enzyme immune assay (EIA). Hydrocorticosterone was quantified using a polyclonal inhouse antibody against cortisol-21-hemisuccinate (HS), coupled with bovine serum albumin (BSA) raised in rabbits, used together with the corresponding 21-HS-steroid coupled to peroxidase (HRP) as label for the EIA. Antibody crossreactivity with different glucocorticoids was as follows: 4pregnen-11a,17,21-triol-3,20-dione (cortisol) 100\%, 4pregnen-11ß,21-diol-3,20-dione (corticosterone) $13.2 \%$, and 4-Pregnen-21-ol-3,20-dione (desoxycorticosterone) $<0.1 \%$, respectively (see Methods in Voigt et al. 2004).

Cortisol standards were prepared, in duplicates of $20 \mu \mathrm{l}$, by $1: 1$ dilutions in assay buffer ranging from 0.2 to $100 \mathrm{pg} / 20 \mu \mathrm{l}$. All the duplicates, together with $100 \mu \mathrm{L}$ cortisol-HRP conjugate in assay buffer $\left(50 \mathrm{mM} \mathrm{Na} 2 \mathrm{HPO}_{4} / \mathrm{Na}_{2} \mathrm{HPO}_{4}, 0.15 \mathrm{M}\right.$ 
Fig. 1 The location of the study sites within Larsmo-Öja (upper) and Vaasa (lower map) study areas. The volume of trees obtained from the Multi-Source National Forest Inventory of the year 2015 is also shown in gray scale, whereas the land areas are delimited by the black line. The inset at the top right of the lower map shows the location of the two study areas (black triangles) in central-western Finland
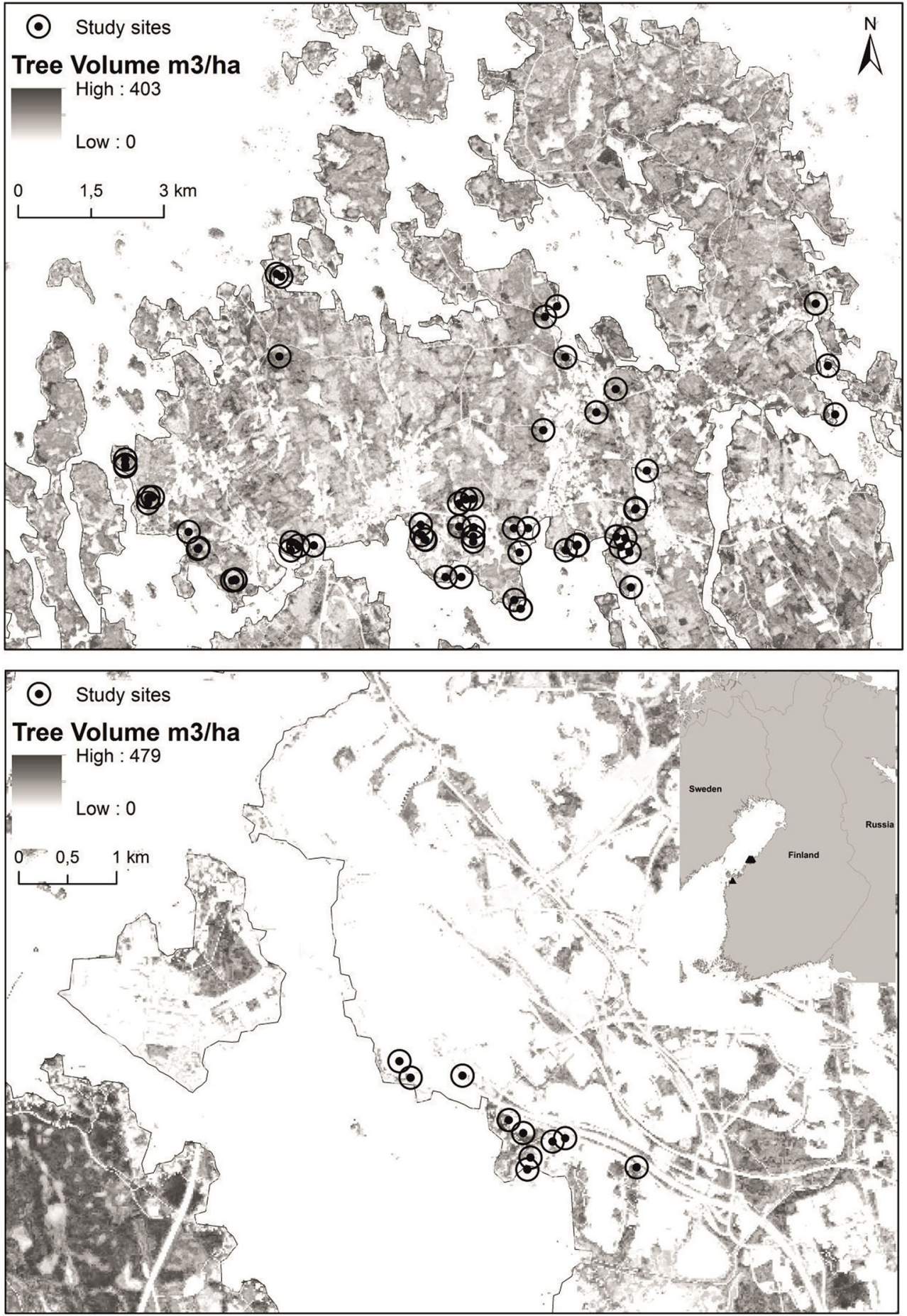

$\mathrm{NaCl}, 0.1 \%$ BSA, pH 7.4), were added to a plate, which was then incubated overnight at $4{ }^{\circ} \mathrm{C}$ and then washed four times with washing solution. One hundred fifty microliter substrate solution was added per well $\left(1.2 \mathrm{mM} \mathrm{H}_{2} \mathrm{O}_{2}, 0.4 \mathrm{mM} \mathrm{3,3',5,5^{ \prime } -}\right.$ tetramethylbenzidine in $10 \mathrm{mM}$ sodium acetate, $\mathrm{pH} 5.5)$, and the plates were incubated in the dark for $40 \mathrm{~min}$. Fifty microliters of $4 \mathrm{~N} \mathrm{H}_{2} \mathrm{SO}_{4}$ were then added to stop the reaction and the samples were measured at $450 \mathrm{~nm}$ with a 12-channel microtiter plate reader (Infinite M 200; Tecan). Hormone concentrations were calculated using the Magellan software (Tecan; see Finkenwirth et al. 2010). The sensitivity of the assay was defined as two standard deviations from the signal given by the zero blank and thus was 0.2 pg per well.

Body condition was calculated for each adult and pup from the mass and femur length measures and using the scaled mass index (Peig and Green 2009). This index scales each individual body mass to the expected value if all individuals had the same body size. The scaled mass index is deemed highly 
robust to measurement errors and allows reliable comparability of body condition from measurements taken on different subjects (Peig and Green 2009). The scaled mass index was calculated separately for adult males and for pups (both sexes combined). It was not possible to calculate body condition of females because the measures were taken during the breeding period, when a fraction of the females may be pregnant with a second litter (Selonen et al. 2016), thereby introducing an unknown bias in the estimation of the real condition. Descriptive statistics of the variables listed above are presented in Supporting Table A1.

\section{Landscape variables}

Our study population inhabits a landscape of fragmented mature forest patches differing in size, shape, and connectedness, and separated by a matrix of commercial monospecific young forest as well as open areas. We classified habitats at and around the study sites into three discrete classes following Wistbacka et al. (2018). A suitable habitat class was represented by layered spruce dominated mixed forests which provide food and shelter and are suitable for breeding. A semi-suitable habitat class depicted mono-specific forests (typically pines) where flying squirrels can move but cannot breed because of lack of some resources (such as food provided by deciduous trees or shelter provided by spruce trees). Unsuitable habitat is represented by open areas covered by young sapling stands or clear-cuts, roads, fields, water, or built up areas, i.e., areas unusable for flying squirrels for moving, feeding, and breeding. These habitats have been mapped in the field and updated yearly (Wistbacka et al. 2018). We measured the cover of each of the three habitat classes within 100 and $200 \mathrm{~m}$ distance from the center of each nesting site (i.e., where the nest boxes are located, following Santangeli et al. (2013b), Wistbacka et al. (2018). These two radii have been previously found to capture habitat within the biologically relevant space used by breeding male and female flying squirrels (Hanski et al. 2000; Santangeli et al. 2013b; Wistbacka et al. 2018). Next, we also calculated the total size of the suitable forest habitat patch where the nesting site is centered. Finally, we considered the perimeter of the patch of suitable forest where the nest was centered. We extracted the amount of this perimeter bordering with semi-suitable habitat. We then calculated the proportion of the total perimeter of the patch of suitable habitat bordering semi-suitable habitat. This variable, hereafter named "embedding," provides a measure of how the focal patch is sheltered by semi-suitable habitat as compared with unsuitable open habitats (see also Wistbacka et al. 2018). Thus, low values of embedding indicate patches that are highly exposed, that is, largely bordering open areas, and that may increase the stress level of flying squirrels living in those, as found in other species (e.g., Brearley et al. 2012). All above variables were extracted in a Geographic Information System (GIS).
Descriptive statistics of the environmental variables listed above are presented in Supporting Table A2.

\section{Social environment variables: relative sex-specific density}

We used all the locations of recorded adult flying squirrels in the study region to build kernel density maps representing the relative density of male and female separately in 2015 and 2016 in ArcGIS 10.1 (CESRI). Next, we extracted the sexspecific and year specific relative density at the location (i.e., nest site) from where we had cortisol data on each adult flying squirrel. We later use this measure of sex-specific relative density as a proxy for intra-sex interactions and competition that may affect cortisol concentration of an individual (Charbonnel et al. 2008). Descriptive statistics of relative congener density are provided in Supporting Table A1.

\section{Statistical analyses}

Basic model structure In order to address each of the study questions (see above), we first set up a basic and minimal general linear mixed model (GLMM) structure including as the response variable the cortisol concentration measured for each individual. We included in this basic model the identity of a family (e.g., a common value for all pups and adults found in the same site and assumed to be related to each other) as a random factor to account for possible pseudo-replication resulting from cortisol values measured from individuals of the same family. Although we attempted to collect as similar amount of hair $(10 \mathrm{mg})$ per individual as possible, some variation in the amount collected was unavoidable. Therefore, we included the micrograms of analyzed hairs (log transformed) as a weight to correct for the fact that samples with a larger amount of hairs are likely to yield more precise measures of cortisol, and thus were given higher weight on the results, compared with samples based on a lower amount of hair. As data were collected across two study years (2015 and 2016) and two rather disjunct study areas (Larsmo/Öja, dominated by managed forests, and Vaasa, a urban recreational parkland area), we also included these two variables as categorical (i.e., area and year; each with two classes) in each model. As area and year are part of the study design, hence, they are a priori included in each model, so that their effect would be controlled for in each case.

Models with predictors We then used the above described basic model structure to test the hypotheses stated in the five aims by adding in turn the relevant covariates. Building a separate model for each of the study questions is appropriate given sample size limitations and collinearity among many of 
the predictors, particularly those referring to landscape (see also Wistbacka et al. 2018). The first model aimed to investigate sex and age variation in cortisol levels (1) by including these two categorical variables to the basic model structure $(n=192)$, as well as their interaction.

Next, we investigated the effects of the environmental covariates (aim 2) on cortisol concentration. Again, we started from the basic model detailed above and added in turn each of the eight landscape predictors, i.e., amount of suitable, semisuitable, and unsuitable habitat, within $100 \mathrm{~m}$ and within $200 \mathrm{~m}$ radius, as well as the size of the forest patch and embedding (sample size $=192$ ). In this model, age (pup versus adult) and sex of the individuals, as well as their interaction, were also included to control for differential responses to habitat by individuals of different sex and age classes. This resulted in eight separate models, each testing the separate effect of each landscape predictor on cortisol concentration.

The impact of conspecific density on cortisol levels (3) was addressed by including the relative congener density as a covariate to the basic model using a subset of adult only data ( $n=31$ and 48 for male and female models respectively). This resulted in two separate models, one for adult males and one for adult females.

Variations in hormone levels according to body condition (4) were considered by adding the scaled body mass index to the basic model using a subset of data from adult males and pups in two separate models ( $n=31$ and 111 respectively). Finally, correlations between females and pups cortisol levels (5) were studied by running two simple linear models with adult female cortisol as the response and either the average cortisol level or the scaled mass index of the pup females from the same litter as the predictor, while controlling for year and study area $(n=26)$. We only considered pup females in the latter model to avoid unnecessary noise in the data caused by the sex ratio, because male pups were found to have higher cortisol concentration than female pups.

The response variable was log-transformed to reduce the spread of the values, whereas all landscape level variables were scaled and centered to zero mean and unit variance to allow for comparability in the estimates of their effect sizes. All analyses were performed in R software version 3.4.3 (R Core Development Team 2017).

\section{Results}

Hair cortisol concentrations in flying squirrels significantly vary by sex and age of individuals, being higher in adults than in pups, and in males compared to females (Table 1; Fig. 2). We found no relationship between cortisol concentrations and any of the landscape variables, such as suitable, semi-suitable, and unsuitable habitat cover at the two spatial scales, as well as no relationship with the size of the forest patch or its embedding
Table 1 Results of separate general linear mixed models testing the effect of age, sex, and their interaction (as per aim 1), as well as the effect of patch size, embedding, and other landscape variables (i.e., suitable, semi-suitable, and unsuitable habitat cover) at two different spatial scales (100 and $200 \mathrm{~m}$ around the site; aim 2) on the cortisol level of individual flying squirrels. See "Methods" for further details on models' structure and description of each variable. The sample size for all these models was 192 units

\begin{tabular}{llllll}
\hline Aim & Variable & Estimate & SE & $t$ & $p$ \\
\hline & Area (Vaasa) & 0.16 & 0.09 & 1.82 & 0.074 \\
& Year (2016) & 0.11 & 0.07 & 1.62 & 0.110 \\
1 & Age (pup) & -0.81 & 0.05 & -15.22 & $<\mathbf{0 . 0 0 1}$ \\
1 & Sex (male) & 0.17 & 0.05 & 3.28 & $\mathbf{0 . 0 0 1}$ \\
1 & Age (pup) * sex (male) & -0.13 & 0.11 & -1.25 & 0.215 \\
2 & Patch size & -0.06 & 0.05 & -1.28 & 0.204 \\
2 & Embedding & 0.03 & 0.03 & 0.92 & 0.357 \\
2 & Suitable100 & 0.05 & 0.03 & 1.48 & 0.143 \\
2 & SemiSuitable100 & 0.00 & 0.03 & 0.01 & 0.994 \\
2 & Suitable200 & 0.01 & 0.04 & 0.22 & 0.824 \\
2 & SemiSuitable200 & 0.00 & 0.03 & 0.01 & 0.994 \\
2 & Unsuitable100 & -0.05 & 0.03 & -1.56 & 0.122 \\
2 & Unsuitable200 & 0.00 & 0.03 & -0.14 & 0.886 \\
\hline
\end{tabular}

Significant variables $(\alpha<0.05)$ are shown in bold font

(Table 1). Moreover, cortisol concentrations of adult male and female flying squirrels were not related to the relative density of other adults of the same sex in the area (Table 2). Cortisol levels of adult males as well as pups of both sexes were not related to their body condition (Table 2). Moreover, we found no clear relationship between the cortisol level of adult female flying squirrels and the average cortisol level or body condition of the female pups from their litter (cortisol level, estimate \pm $\mathrm{SE}=-0.72 \pm 0.35 ; t=-2.04 ; p=0.054$; and body condition, $0.73 \pm 0.56 ; t=1.30 ; p=0.21$ ).

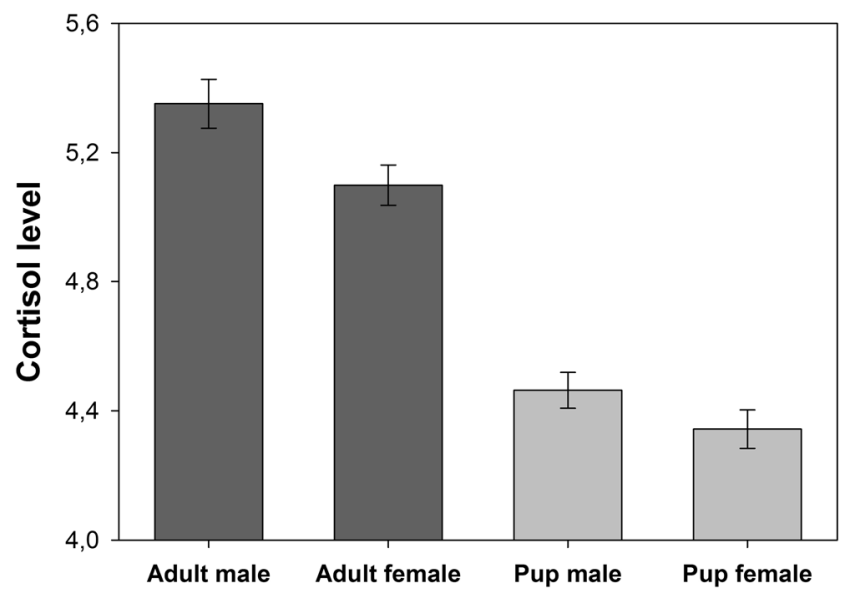

Fig. 2 Cortisol concentrations (pg/mg of hairs on the log scale) of flying squirrels in relation to age and sex. Bars represent least square means and standard errors for the cortisol derived from the relative models presented in Table 1 which also included the interaction term between sex and age 
Table 2 Results of separate general linear mixed models testing the effect of the relative density of individuals of the same sex separately for adult male and female flying squirrels (as per aim 3), as well as the effect of body condition of males and pups (aim 4) on the cortisol level of individual flying squirrels. See "Methods" for further details on models' structure. The sample size for models of study aim 3 was 31 for males and 48 for females, and for aim 4 was 31 and 111 for males and pups, respectively

\begin{tabular}{clllll}
\hline Study aim & Variable & Estimate & SE & $t$ & $p$ \\
\hline 3 & Congener density, males & 0.67 & 0.33 & 2.01 & 0.182 \\
3 & Congener density, females & -0.13 & 0.17 & -0.75 & 0.493 \\
4 & Body condition, males & 0.00 & 0.01 & -0.39 & 0.731 \\
4 & Body condition, pups & 0.00 & 0.00 & 0.07 & 0.946 \\
\hline
\end{tabular}

\section{Discussion}

\section{Effects of sex and age on cortisol concentrations}

The higher cortisol concentration found in adult male flying squirrels, compared with females, contradicts our initial prediction based on previous studies (e.g., Reeder and Kramer 2005; Waterhouse et al. 2017). This result may be explained by the male flying squirrels having larger and more widely overlapping home ranges than females (Selonen and Hanski 2003; Selonen et al. 2013), and by their breeding strategy. The flying squirrel, as many other arboreal squirrels, departs from the general pattern found in mammals whereby males are larger than females (Selonen et al. 2016). Moreover, males typically compete for females during the breeding season, and this competition was identified as a potential main driver of body mass variation in male flying squirrels (Selonen et al. 2016). Conversely, adult female flying squirrels can also frequently occupy the same nesting site in winter, suggesting they are somewhat more social, at least during winter, than males (Selonen et al. 2014). Possibly, competition and the higher vagility of male compared with female flying squirrels may have repercussions at the physiological level, ultimately affecting the chronic stress of individuals. This topic requires further empirical studies before solid conclusions are drawn.

The finding that flying squirrel adults have higher cortisol concentrations than pups may also relate to the life stage and activity patterns relative to the period when cortisol concentrations accumulate in the hairs (i.e., the growth time of sampled hair). During this reproductive period, adult females may need to increase their foraging activity and males may move across large areas while seeking for receptive females (Selonen et al. 2013; Selonen et al. 2016). Conversely, pups spend most of the time in the nest box during the same period and may be less affected by external stressors, e.g., landscape and predators, thereby showing lower cortisol concentrations despite potential competition among siblings, which is largely unknown.

\section{Effects of environmental factors on cortisol concentrations}

We found no indication that any of the landscape factors considered would have a measurable effect on hair cortisol concentration of flying squirrels. The broad assumption was that this forest dwelling species typically prefers to move within mature forests and avoids crossing large gaps as this may expose it to predation and other risks (Mäkelainen et al. 2016). However, especially male flying squirrels have been found to cross small forest gaps (Selonen and Hanski 2003). Gap crossing in search of food resources and nesting cavities may be common when individuals settle in small forest patches and areas with limited amounts of suitable habitat (Mäkeläinen et al. 2016; Selonen and Hanski 2003). The landscape variables (e.g., amount of suitable habitat and patch size) and spatial scale (100 and $200 \mathrm{~m}$ radii) considered here have been found to have a strong effect on overall, and especially of breeding females, occurrence in flying squirrels in Finland (Jokinen et al. 2014; Santangeli et al. 2013b; Wistbacka et al. 2018). However, despite the importance of these landscape features for flying squirrel breeding habitat choice, they did not have any effect on hair cortisol concentration, suggesting that there might be different factors affecting habitat selection versus physiological responses in this study system and species. Habitat choice by flying squirrels is largely driven by resource availability (such as food and cavities for breeding) as well as predator avoidance (such as Ural owl Strix uralensis and eagle owl Bubo bubo, Byholm et al. 2012). These avian predators are however rather scarce in the study area (about five resident pairs of eagle owl in Larsmo/Öja and none in Vaasa, whereas Ural owl is very scarce in both areas; authors own observations). Therefore, predation risk may represent only a negligible source of stress for flying squirrels locally. This could thereby explain the lack of a strong physiological response to landscape-mediated factors.

In addition to natural environmental stressors, like food and predators, also anthropic presence could lead to a stress response in wild animals. A study from Australia reported an effect of habitat edges bordering major roads on the hair cortisol concentration of the squirrel glider (Petaurus norfolcensis) in an urban environment (Brearley et al. 2012). In that case, the habitat-mediated effect was direct, as the species was found to avoid edge areas due to anthropogenic noise and disturbance (Brearley et al. 2010). More generally, a meta-analysis study found a broadly consistent positive link between glucocorticoid levels and anthropogenic disturbance (Dantzer et al. 2014). Overall, our results reinforce the broad view that stress responses to environmental disturbances in wild animals may often be contextdependent and species-specific (see e.g., Brearley et al. 2012; Romero and Wikelski 2002). 
Finally, the results could be partially masked by methodological constraints. In this study, we were only able to measure cortisol concentrations through the non-invasive collection and analyses of hair samples from the tail of the individuals. It has been shown that the body location from which hair samples are collected, as well as the type of samples (e.g., faces versus hair) may affect the resulting stress values (Mastromonaco et al. 2014; Terwissen et al. 2013). Moreover, we collected hair samples that are representative of the breeding period of the species. It is possible that cortisol concentrations in this species may be elevated during breeding to a level that could mask the effect of any other external factors, such as landscape-mediated factors. Therefore, collecting samples from different seasons, different parts of the body as well as different sample types would represent a step forward toward ultimately concluding whether landscape features have any effect on the chronic stress of flying squirrels. In addition, North American flying squirrels have been recently shown to have low sensitivity to cortisol (Desantis et al. 2018). This may be the case also for the Siberian flying squirrel, thereby explaining the findings of this study. This issue should however be investigated in depth with further studies.

\section{Effects of social and intrinsic factors on cortisol}

The result that congener density was not related to cortisol concentration in adult flying squirrels contrasts with our initial prediction that individuals living in high density areas may show higher stress response. This pattern has been uncovered in several mammal species and may relate to intra-specific competition (Dantzer et al. 2013; Mastromonaco et al. 2014). In the case of the flying squirrel population studied here, it may be possible that densities may not be high enough (Santangeli et al. 2013a) to trigger a measurable physiological response. Also, our measure of relative density represents only a crude proxy of real densities because not all the study area and all adult flying squirrels are extensively surveyed. This may have caused an underestimation of the real densities, thereby potentially masking a density-dependent effect on cortisol concentrations measured. It may also be possible that cortisol concentrations in hairs may not show as strong signal of chronic stress as it may be from facial samples, as the findings from a study on a wild population of chipmunks (Tamias striatus) suggests (Mastromonaco et al. 2014).

We did not find any relationship between hair cortisol concentrations and body condition in adult male flying squirrels, nor a link between cortisol concentrations of mothers with body condition of their pups. There was however a tendency for female pups to have similar cortisol concentrations as their mothers. Early life stress in rodent pups has been associated with the level of parental care and stress of mothers (Dantzer et al. 2013). For example, pups from less caring mothers and those that have been longer separated from their mothers were found to have higher stress levels (Novais et al. 2017). In the case of flying squirrels, adult chronic stress, as measured by hair cortisol concentrations, does not appear to be related by the physical or social landscape around them, which may also explain the lack of a relationship between cortisol concentrations in mothers with cortisol, as well as the body condition, of their pups. This lack of strong correlation could however be due to the impossibility to control for female body size on neither their cortisol level, nor the level of their pups, due to possible unknown pregnancy of the females which could bias the observed body condition. Previous evidence indeed suggests body mass to be lower in chronically stressed individuals due to inhibition of their growth hormones under stressful conditions (Breuner et al. 2013), and during adulthood, chronic stress may cause a reduction in body mass (Hodges et al. 2006). However, all the above patterns appear to be species and context specific (Breuner et al. 2013), and the results presented here seem to confirm that broad conclusion.

\section{Conclusions}

The limited sample size in view of the large variation in the variables measured may have led to a type II error regarding the potential effect of the environmental variables on flying squirrel cortisol concentration. As for many field studies on elusive nocturnal species, logistical constraints hindered the collection of a larger amount of data than that used for this study. Enlarging the sample size might help to conclude if the lack of any relationship between cortisol concentration and the environmental variables tested is genuine or might have been due to the limited sample size. Ultimately, and keeping in mind the above limitations, our findings suggest that while hair cortisol concentrations of flying squirrels differ by age and sex classes, they appear to be not affected by landscape (e.g., amount of habitat and its fragmentation) or social (congener density) factors, nor by intrinsic factors (e.g., body condition). From a conservation perspective, this seems to indicate that habitat amount, while being a strong determinant of the occurrence of the species (Santangeli et al. 2013b; Wistbacka et al. 2018), may not measurably affect the chronic stress of individuals. Ultimately, these results may represent only a first step toward understanding stress, and the drivers of it, in the flying squirrel. Future work should focus on validating the use of hair cortisol concentrations as a measure of stress (Mastromonaco et al. 2014) in this species. In addition, more empirical, and possibly experimental work is needed to uncover the link between chronic stress and several intrinsic factors relating to individual flying squirrel health, life history stage, and social status, as well as extrinsic factors relating to environmental conditions, including both landscape and predation risk. 
Acknowledgments We thank Arild Husby for funding the cortisol analyses and M. Dehnhard for running the lab analyses; Sarah Knowles for help with field equipment; Ben Dantzer for useful feedback; and numerous field-assistants who helped collecting the data; E. Mori and four other reviewers provided useful comments that helped improve the manuscript. RW thanks Svensk-Österbottniska samfundet, Societas Pro Fauna et flora Fennica, Vuokon Luonnonsuojelusäätiö, and Suomen Luonnonsuojelun säätiö.

Funding information Open access funding provided by University of Helsinki including Helsinki University Central Hospital. AS thanks the Academy of Finland (grant no. 307909) and Kone Foundation for financial support of this study.

Compliance with ethical standards The work has been carried out under the ethical standards of the Finnish animal experimentation committee and international standards. The capturing and handling of flying squirrels was done under the permits issues by the local environmental center (N. EPOELY/412/07.01/2012 and N. EPOELY/321/2017).

Conflict of interest The authors declare no financial or non-financial conflicts of interest related to this work.

Open Access This article is distributed under the terms of the Creative Commons Attribution 4.0 International License (http:// creativecommons.org/licenses/by/4.0/), which permits unrestricted use, distribution, and reproduction in any medium, provided you give appropriate credit to the original author(s) and the source, provide a link to the Creative Commons license, and indicate if changes were made.

\section{References}

Bairos-Novak KR, Ryan CP, Freeman AR, Anderson WG, Hare JF (2018) Like mother, like daughter: heritability of female Richardson's ground squirrel Urocitellus richardsonii cortisol stress responses. Curr Zool 64:153-163

Brearley G, Bradley A, Bell S, McAlpine C (2010) Influence of contrasting urban edges on the abundance of arboreal mammals: a study of squirrel gliders (Petaurus norfolcensis) in southeast Queensland, Australia. Biol Conserv 143:60-71

Brearley G, McAlpine C, Bell S, Bradley A (2012) Influence of urban edges on stress in an arboreal mammal: a case study of squirrel gliders in southeast Queensland, Australia. Landsc Ecol 27:1407-1419

Breuner CW, Delehanty B, Boonstra R (2013) Evaluating stress in natural populations of vertebrates: total CORT is not good enough. Funct Ecol 27:24-36

Byholm P, Burgas D, Virtanen T, Valkama J (2012) Competitive exclusion within the predator community influences the distribution of a threatened prey species. Ecology 93:1802-1808

Charbonnel N, Chaval Y, Berthier K, Deter J, Morand S, Palme R, Cosson JF (2008) Stress and demographic decline: a potential effect mediated by impairment of reproduction and immune function in cyclic vole populations. Physiol Biochem Zool 81:63-73

Chen HL, Koprowski JL (2016) Barrier effects of roads on an endangered forest obligate: influences of traffic, road edges, and gaps. Biol Conserv 199:33-40

Creel S, Fox JE, Hardy A, Sands J, Garrott B, Peterson RO (2002) Snowmobile activity and glucocorticoid stress responses in wolves and elk. Conserv Biol 16:809-814

Dantzer B, Newman AEM, Boonstra R, Palme R, Boutin S, Humphries MM, McAdam AG (2013) Density triggers maternal hormones that increase adaptive offspring growth in a wild mammal. Science 340: 1215-1217

Dantzer B, Fletcher QE, Boonstra R, Sheriff MJ (2014) Measures of physiological stress: a transparent or opaque window into the status, management and conservation of species? Conserv Physiol 2(1): cou023. https://doi.org/10.1093/conphys/cou023

Desantis LM, Bowman J, Lahoda CV, Boonstra R, Burness G (2016) Responses of new world flying squirrels to the acute stress of capture and handling. J Mammal 97:80-88

Desantis LM, Bowman J, Vijayan MM, Burness G (2018) Seasonal changes in acute stressor-mediated plasma glucocorticoid regulation in new world flying squirrels. Gen Comp Endocrinol 266:78-86

Ewers RM, Didham RK (2006) Confounding factors in the detection of species responses to habitat fragmentation. Biol Rev 81:117-142

Finkenwirth C, Jewgenow K, Meyer HHD, Vargas A, Dehnhard M (2010) PGFM (13,14-dihydro-15-keto-PGF2a) in pregnant and pseudo-pregnant Iberian lynx: a new noninvasive pregnancy marker for felid species. Theriogenology 73:530-540

Finnish Forest Research Institute (2014) Finnish Statistical Yearbook of Forestry:2014 Available at: http://www.metla.fi/julkaisut/ metsatilastollinenvsk/index-en.htm

Gorini L, Linnell JDC, May R, Panzacchi M, Boitani L, Odden M, Nilsen EB (2012) Habitat heterogeneity and mammalian predator-prey interactions. Mammal Rev 42:55-77

Hanski IK, Stevens PC, Ihalempiä P, Selonen V (2000) Home-range size, movements, and nest-site use in the Siberian flying squirrel, Pteromys volans. J Mammal 81:798-809

Hodges KE, Boonstra R, Krebs CJ (2006) Overwinter mass loss of snowshoe hares in the Yukon: starvation, stress, adaptation or artefact. J Anim Ecol 75:1-13

Huhman KL, Solomon MB, Janicki M, Harmon AC, Lin SM, Israel JE, Jasnow AM (2003) Conditioned defeat in male and female Syrian hamsters. Horm Behav 44:293-299

Jokinen M, Mäkeläinen S, Ovaskainen O (2014) 'Strict', yet ineffective: legal protection of breeding sites and resting places fails with the Siberian flying squirrel. Anim Conserv 18:167-175

Koprowski JL (2005) The response of tree squirrels to fragmentation: a review and synthesis. Anim Conserv 8:369-376

Koskimäki J, Huitu O, Kotiaho JS, Lampila S, Mäkelä A, Sulkava R, Mönkkönen M (2014) Are habitat loss, predation risk and climate related to the drastic decline in a Siberian flying squirrel population? A 15-year study. Popul Ecol 56:341-348

Lampila S, Wistbacka R, Mäkelä A, Orell M (2009) Survival and population growth rate of the threatened Siberian flying squirrel (Pteromys volans) in a fragmented forest landscape. Ecoscience 16:66-74

Mäkelä A (1996) The occurrence of flying squirrel (Pteromys volans) in the study areas of Alavus during mappings in 1981 and 1994. The flying squirrel in Finland. Report nr 8. WWF Finland

Mäkeläinen S, de Knegt HJ, Ovaskainen O and Hanski IK (2016) Homerange use patterns and movements of the Siberian flying squirrel in urban forests: effects of habitat composition and connectivity. Mov Ecol 4:5. doi: https://doi.org/10.1186/s40462-016-0071-z. eCollection 2016

Mastromonaco GF, Gunn K, McCurdy-Adams H, Edwards DB, SchulteHostedde AI (2014) Validation and use of hair cortisol as a measure of chronic stress in eastern chipmunks (Tamias striatus). Conserv Physiol 2(1):cou055. doi: https://doi.org/10.1093/conphys/cou055. eCollection 2014

Navarro-Castilla Á, Mata C, Ruiz-Capillas P, Palme R, Malo JE, Barja I (2014) Are motorways potential stressors of roadside wood mice (Apodemus sylvaticus) populations? PLoS One 9:e91942

Newton I (1998) Population limitation in birds. Academic Press, San Diego

Nikicicz H, Kasprzak A, Malendowicz LK (1984) Sex differences in adrenocortical structure and function. XIII stereologic studies on 
adrenal cortex of maturing male and female hamsters. Cell Tissue Res 235:459-462

Novais A, Monteiro S, Roque S, Correia-Neves M, Sousa N (2017) How age, sex and genotype shape the stress response. Neurobiol Stress 6:44-56

Peig J, Green AJ (2009) New perspectives for estimating body condition from mass/length data: the scaled mass index as an alternative method. Oikos 118:1883-1891

Pimm SL, Russell GJ, Gittleman JL, Brooks TM (1995) The future of biodiversity. Science 269:347-350

R Core Development Team (2017) R: a language and environment for statistical computing. Version 3.4.3. R foundation for statistical computing. URL https://www.R-project.org/, Vienna, Austria

Rakotoniaina JH, Kappeler PM, Kaesler E, Hämäläinen AM, Kirschbaum C, Kraus C (2017) Hair cortisol concentrations correlate negatively with survival in a wild primate population. BMC Ecol 17:30. https://doi.org/10.1186/s12898-017-0140-1

Reeder DM, Kramer KM (2005) Stress in free-ranging mammals: integrating physiology, ecology, and natural history. J Mammal $86: 225-235$

Romero LM, Wikelski M (2002) Exposure to tourism reduces stressinduced corticosterone levels in Galápagos marine iguanas. Biol Conserv 108:371-374

Santangeli A, Hakkarainen H, Laaksonen T, Korpimaki E (2012) Home range size is determined by habitat composition but feeding rate by food availability in male Tengmalm's owls. Anim Behav 83:1115-1123

Santangeli A, Hanski IK, Makela H (2013a) Integrating multi-source forest inventory and animal survey data to assess nationwide distribution and habitat correlates of the Siberian flying squirrel. Biol Conserv 157:31-38

Santangeli A, Wistbacka R, Hanski IK, Laaksonen T (2013b) Ineffective enforced legislation for nature conservation: a case study with Siberian flying squirrel and forestry in a boreal landscape. Biol Conserv 157:237-244

Sapolsky RM, Romero LM, Munck AU (2000) How do glucocorticoids influence stress responses? Integrating permissive, suppressive, stimulatory, and preparative actions. Endocr Rev 21:55-89

Selonen V, Hanski IK (2003) Movements of the flying squirrel Pteromys volans in corridors and in matrix habitat. Ecography 26:641-651

Selonen V, Painter JN, Rantala S, Hanski IK (2013) Mating system and reproductive success in the Siberian flying squirrel. J Mammal 94: $1266-1273$

Selonen V, Hanski IK, Wistbacka R (2014) Communal nesting is explained by subsequent mating rather than kinship or thermoregulation in the Siberian flying squirrel. Behav Ecol Sociobiol 68:971-980

Selonen V, Wistbacka R, Santangeli A (2016) Sex-specific patterns in body mass and mating system in the Siberian flying squirrel. BMC Zoology 1:9. https://doi.org/10.1186/s40850-016-0009-3
Sheriff MJ, Krebs CJ, Boonstra R (2009) The sensitive hare: sublethal effects of predator stress on reproduction in snowshoe hares. J Anim Ecol 78:1249-1258

Sheriff MJ, Krebs CJ, Boonstra R (2010) The ghosts of predators past: population cycles and the role of maternal programming under fluctuating predation risk. Ecology 91:2983-2994

Sheriff MJ, Dantzer B, Delehanty B, Palme R, Boonstra R (2011) Measuring stress in wildlife: techniques for quantifying glucocorticoids. Oecologia 166:869-887

Shero MR, Krotz RT, Costa DP, Avery JP, Burns JM (2015) How do overwinter changes in body condition and hormone profiles influence Weddell seal reproductive success? Funct Ecol 29:1278-1291

Smith WP (2012) Sentinels of ecological processes: the case of the northern flying squirrel. BioScience 62:950-961

Sutherland WJ, Pullin AS, Dolman PM, Knight TM (2004) The need for evidence-based conservation. Trends Ecol Evol 19:305-308

Tarjuelo R, Barja I, Morales MB, Traba J, Benítez-López A, Casas F, Arroyo B, Delgado MP, Mougeot F (2015) Effects of human activity on physiological and behavioral responses of an endangered steppe bird. Behav Ecol 26:828-838

Terwissen CV, Mastromonaco GF, Murray DL (2013) Influence of adrenocorticotrophin hormone challenge and external factors (age, sex, and body region) on hair cortisol concentration in Canada lynx (Lynx canadensis). Gen Comp Endocrinol 194:162-167

Trapp SE, Day CC, Flaherty EA, Zollner PA, Smith WP (2019) Modeling impacts of landscape connectivity on dispersal movements of northern flying squirrels (Glaucomys sabrinus griseifrons). Ecol Model 394:44-52

Voigt CC, Fassbender M, Dehnhard M, Wibbelt G, Jewgenow K, Hofer H, Schaub GA (2004) Validation of a minimally invasive bloodsampling technique for the analysis of hormones in domestic rabbits, Oryctolagus cuniculus (Lagomorpha). Gen Comp Endocrinol 135(2004):100-107

Waterhouse MD, Sjodin B, Ray C, Erb L, Wilkening J, Russello MA (2017) Individual-based analysis of hair corticosterone reveals factors influencing chronic stress in the American pika. Ecol Evol 7: 4099-4108

Wistbacka R, Orell M, Santangeli A (2018) The tragedy of the sciencepolicy gap - revised legislation fails to protect an endangered species in a managed boreal landscape. For Ecol Manag 422:172-178

Publisher's note Springer Nature remains neutral with regard to jurisdictional claims in published maps and institutional affiliations. 